Ana Cláudia Alves Leão?

(ㄱ) https://orcid.org/0000-0003-2581-8581

Nayra Suze Souza e Silva²

Ohttps://orcid.org/0000-0002-8420-0827

Romerson Brito Messias 3

O hitps://orcid.org/0000-0002-4781-5050

Desirée Sant'Ana Haikal²

(- https://orcid.org/0000-0002-0331-0747

Marise Fagundes Silveira 2,4

Dhttps://orcid.org/0000-0002-8821-3160

Lucineia de Pinho ${ }^{4}$

Ohttps:///orcid.org/0000-0002-2947-5806

Rosângela Ramos Veloso Silva ${ }^{4}$

O https://orcid.org/0000-0003-3329-8133

Maria Fernanda Santos Figueiredo

Brito $^{4}$

Cohttps://orcid.org/0000-0001-5395-9491

\section{Consumo de álcool em professores da rede pública estadual durante a pandemia da COVID-19}

\author{
Alcohol consumption in state public school teachers \\ during the COVID-19 pandemics
}

DOI: 10.1590/0047-2085000000368

\section{RESUMO}

Objetivo: Avaliar o consumo de álcool entre os professores da rede pública estadual do ensino básico antes e durante a pandemia da COVID-19 e os fatores associados aos que aumentaram o consumo de bebidas alcoólicas durante a pandemia. Métodos: Inquérito epidemiológico do tipo websurvey, realizado com professores da educação básica das escolas da rede pública estadual de Minas Gerais. A coleta ocorreu de agosto a setembro/2020 via formulário digital e contou com o apoio da Secretaria de Estado de Educação. A variável dependente adotada foi o aumento no consumo de bebidas alcoólicas pelos professores durante a pandemia. Foi utilizada a regressão de Poisson, adotando nível de significância de 5\%. Resultados: Participaram do estudo 15.641 professores de 795 municípios mineiros. Em relação ao consumo de bebidas alcoólicas, antes da pandemia, 46,9\% dos professores consumiam pelo menos uma vez por semana e durante a pandemia 7,1\% relataram que estavam bebendo mais do que costumavam. Observou-se maior consumo de bebida alcoólica durante a pandemia entre os homens, com menor faixa etária, com maior renda familiar, os que tiveram muita dificuldade em realizar as atividades escolares, os insatisfeitos com o trabalho, os que começaram a ter problemas de sono e naqueles com algum familiar/amigo que desenvolveu sintomas graves da COVID-19. Conclusões: Os resultados evidenciaram que a maioria dos participantes manteve o seu consumo ou o diminuiu durante a pandemia, com 7,1\% aumentando o uso de álcool. Esse aumento foi associado a fatores sociodemográficos e econômicos, condições de trabalho durante a pandemia, comportamentos/hábitos de vida e condições de saúde.

\section{PALAVRAS-CHAVE}

COVID-19, pandemia, professores, bebidas alcoólicas, saúde.

\begin{abstract}
Objective: To evaluate alcohol consumption among state public elementary school teachers before and during the COVID-19 pandemic and the factors associated with who increased their alcohol consumption during the pandemic. Methods: Websurvey-type epidemiological survey, carried out with primary education teachers from public schools in Minas Gerais. A probabilistic sample with proportionality for state teachers was estimated. The collection took place from August to September 2020 via digital form and had the support of the State Department of Education. The dependent variable adopted was the increase in the consumption of alcoholic beverages by teachers during the pandemic. Poisson Regression was used, adopting a significance level of 5\%. Results: 15,641 teachers from 795 Minas Gerais municipalities participated in the study. Regarding the consumption of alcoholic beverages, before the pandemic $46.9 \%$ of teachers consumed at least once a week and during the pandemic $7.1 \%$ reported that they were drinking more than they used to. Higher consumption of alcoholic beverages was observed during the pandemic among men, with a lower age group, higher family income, those who had great difficulty to carry out school activities, those dissatisfied with work, those who started having sleep problems and in those where a family member or friend developed symptoms serious problems of COVID-19. Conclusions: The results showed that most participants maintained their consumption or decreased it during the pandemic. This increase was associated with sociodemographic and economic factors, working conditions during the pandemic, behaviors/life habits and health conditions.
\end{abstract}

\section{KEYWORDS}

COVID-19, pandemic, teachers, alcoholic beverages, health.
Received in: 14/Apr/2021. Approved in: 29/Dec/2021

1 Hospital Universitário Clemente de Faria da Universidade Estadual de Montes Claros (Unimontes), Montes Claros, MG, Brasil.

2 Programa de Pós-graduação em Ciências da Saúde da Universidade Estadual de Montes Claros (Unimontes), Montes Claros, MG, Brasil.

3 Departamento de Saúde Mental e Coletiva da Universidade Estadual de Montes Claros (Unimontes), Montes Claros, MG, Brasil.

4 Programa de Pós-graduação em Cuidado Primário em Saúde da Universidade Estadual de Montes Claros (Unimontes), Montes Claros, MG, Brasil.

Address for correspondence: Ana Cláudia Alves Leão. Avenida Rui Braga, s/n, Vila Mauricéia - 39401-089 - Montes Claros, MG, Brasil.

E-mail:a.claudialeao@hotmail.com 


\section{INTRODUÇÃO}

Diante da pandemia da COVID-19, a literatura nacional e internacional relata mudanças em relação ao consumo de bebidas alcoólicas na população ${ }^{1-4}$. Entre essas mudanças, está presente o aumento no consumo de álcool ${ }^{1,3,5,6}$. Consumir bebidas alcoólicas excessivamente representa um grave problema de saúde pública, com potenciais efeitos socioeconômicos e sobre a saúde física e mental dos indivíduos ${ }^{7}$. Considerando os problemas decorrentes da pandemia, como o distanciamento social, o aumento no consumo de bebidas alcoólicas ficou ainda mais proeminente e prejudicial ${ }^{3,5}$.

O consumo de álcool encontra-se associado a condições culturais e sociais em todo o mundo, muitas vezes de forma recreacional e com o objetivo de alívio de sintomas físicos e mentais estressantes do cotidiano. No mundo, antes da pandemia, a prevalência estimada entre a população adulta para o abuso episódico do álcool foi de 18,4\%, sendo as regiões europeias as que tiveram as maiores prevalências. As taxas de mortalidade atribuíveis ao álcool foram de 33 mortes por 100.000 pessoas $^{7}$. O consumo excessivo episódico de álcool entre jovens brasileiros era de 17\% em 2013, apresentando diferenças regionais ${ }^{8}$.

No cenário da pandemia da COVID-19, um estudo realizado em 83 países demonstrou que 36\% dos participantes relataram aumento no uso de álcool2. No Brasil, nesse mesmo cenário, o aumento do consumo de álcool ocorreu em 17,6\% da população adulta'. Estudos também apresentaram resultados acerca da redução e/ou estado inalterado do uso de álcool na pandemia da COVID-193,6. Estudo com populações da América Latina e do Caribe reforça esses dados, com 33,3\% relatando diminuição do consumo ${ }^{3}$. Na Alemanha, 19,4\% da população apresentou menor consumo e 37,7\% não teve alteração do consumo ${ }^{6}$.

As medidas de isolamento e distanciamento social durante a pandemia, com aumento das atividades realizadas em ambiente familiar, nova mudança de perspectiva laboral e estudantil, convívio mais intenso com familiares e problemas domésticos, acabaram por se tornar eventos estressores, predisponentes ao abuso alcoólico 9 . Nesse cenário, observa-se o aumento do consumo de bebidas alcoólicas em ambiente domiciliar ${ }^{10}$, encontrando-se a classe dos professores entre as categorias profissionais de risco para maior incidência de distúrbios relacionados à saúde mental e, consequentemente, para aumento do consumo do álcool'1.

Os professores, na atual pandemia, foram submetidos a desafios marcantes no ensino, tais como ensino remoto, aulas on-line, novas plataformas e estratégias pedagógicas, associados à mudança do local de trabalho da instituição de ensino para o ambiente doméstico. Nessa perspectiva, os eventos estressores influenciaram a saúde mental e os hábitos dessa classe profissional'".
Nesse sentido, os professores podem buscar no álcool uma fonte de alívio para as situações adversas iniciadas com a pandemia. Há carência de trabalhos associando a readaptação funcional dos docentes da educação básica ao uso/ abuso dessa substância, especialmente no estado de Minas Gerais. Diante disso, este estudo tem como objetivo avaliar o consumo de álcool entre os professores da rede pública estadual do ensino básico antes e durante a pandemia da COVID-19, assim como os fatores associados aos professores que aumentaram o consumo de bebidas alcoólicas durante a pandemia.

\section{MÉTODOS}

\section{Desenho do estudo}

Este estudo faz parte do Projeto ProfSMoc - Etapa Minas Covid "Condições de saúde e trabalho entre professores da rede estadual de ensino do estado de Minas Gerais na pandemia da COVID-19". Trata-se de um inquérito epidemiológico do tipo websurvey, realizado com professores da educação básica das escolas da rede pública estadual de ensino do estado de Minas Gerais, Brasil. O estado de Minas Gerais possui aproximadamente 90 mil professores da educação básica (dado fornecido pela Secretaria de Estado de Educação de Minas Gerais - SEE-MG - mediante folha de pagamento do mês de julho de 2020), atuantes em 3.441 escolas públicas presentes no estado ${ }^{12}$. Por se tratar de um estudo de websurvey, foram seguidas as recomendações do Checklist for Reporting Results of Internet E-Surveys ${ }^{13}$.

\section{Cálculo amostral}

Para o cálculo amostral, foi utilizada fórmula considerando populações infinitas. Foi considerada uma prevalência de 50\% com a intenção de obter o maior tamanho amostral e, consequentemente, o maior poder de inferência para diferentes variáveis. O erro tolerável adotado foi de $3 \%$. Além disso, a amostra foi duplicada $($ deff $=2)$, pois a coleta foi proveniente de conglomerados. Também foi realizado acréscimo de $20 \%$ no tamanho amostral para compensar possíveis perdas (taxa de não resposta), que poderiam comprometer a validade do estudo. Desse modo, estimou-se amostra mínima de 2.564 professores para garantir a representatividade para todo estado de Minas Gerais.

Organizacionalmente, o estado de Minas Gerais é dividido em seis polos, e cada polo é subdividido em Superintendências Regionais de Ensino (SREs). Ao todo, o estado apresenta 45 SREs. O acesso ao quantitativo de professores e à qual SRE o professor estava vinculado foi disponibilizada pela SEE-MG. 


\section{Procedimentos e instrumentos}

Antes de se iniciar a coleta de dados, foram obtidas autorizações por parte da SEE-MG e das 45 SREs. Estudo-piloto prévio foi realizado com 20 professores de cinco diferentes cidades de Minas Gerais para teste e acerto do instrumento de coleta de dados. A coleta de dados ocorreu de 20 de agosto a 11 de setembro de 2020, por meio de formulário digital disponibilizado aos professores via plataforma Google Forms. O link do formulário digital foi amplamente divulgado e enviado para o e-mail institucional de todos os professores do estado. Para evitar o preenchimento automático do formulário por sistemas computacionais, foi utilizado um reCAPTCHA, que apresentava testes em imagens, impedindo que o formulário fosse enviado com sucesso por algum robô. Todas as questões do formulário foram de preenchimento obrigatório, minimizando perdas de informações. O estudo também garantiu o anonimato dos professores, e o formulário de coleta de dados consumia aproximadamente 25 minutos para ser preenchido.

Foram incluídos no estudo os professores em exercício da função docente no ano de 2020, os atuantes na educação infantil, ensino fundamental e/ou ensino médio (vinculados a alguma escola pública estadual de Minas Gerais) e aqueles que aceitassem participar da pesquisa. Não participaram da pesquisa os professores em desvio da função docente, os aposentados e aqueles que responderam "não" quando perguntados se aceitavam participar do estudo.

Este estudo foi conduzido adotando-se como variável dependente o consumo de bebidas alcoólicas, sendo estimada por meio da questão: "Durante a pandemia: a) não consumia bebida alcoólica antes da pandemia e não estou consumindo durante. b) não estou consumindo bebida alcoólica. c) estou bebendo menos do que costumava. d) continuo bebendo com a mesma frequência. e) estou bebendo mais do que costumava. f) tinha parado de beber, mas comecei a beber novamente.". Para analisar o aumento de consumo de bebida alcoólica durante a pandemia, foram consideradas as alternativas "e" e "f". Também foram apresentados o consumo de bebidas alcoólicas antes da pandemia pelos professores, por meio das questões: "Antes da pandemia da COVID-19, quantos dias da semana você consumia bebidas alcoólicas?" e "Antes da pandemia da COVID-19, quantas doses de bebida alcoólica você consumia por dia da semana?".

As variáveis independentes foram organizadas em blocos de assunto: "Perfil sociodemográfico e econômico", "Condições de trabalho durante a pandemia", "Comportamentos/hábitos de vida durante a pandemia" e "Condições de saúde durante a pandemia".

No bloco perfil sociodemográfico e econômico, estão as variáveis: sexo (feminino; masculino), idade em anos (60 ou mais; 41 a 59; até 40), renda familiar em salários mínimos (1 a 2; 3 a 5; 6 a 9; 10 ou mais) (foi considerado o salário mínimo vigente, de R\$1.045,00, na época da coleta de dados) e situação conjugal (com cônjuge; sem cônjuge).
Para as condições de trabalho durante a pandemia, foram abordadas as variáveis: realização de trabalho remoto (sim; não), dificuldade de realizar as atividades (nenhuma dificuldade; pouca; moderada; muita) e satisfação com o trabalho (satisfeito; nem satisfeito nem insatisfeito; insatisfeito).

O bloco de comportamentos/hábitos de vida durante a pandemia foi composto por: adesão ao distanciamento social (sim, totalmente; sim, parcialmente; não), tabagismo (não; ex-fumante; sim), desejo de cuidar da aparência física (aumentou; permaneceu o mesmo; diminuiu), alimentação (melhor padrão alimentar; pior padrão alimentar), prática de atividade física (sim; não) e atividades de lazer (não estava realizando atividades de lazer; diminuiu; não se alterou; aumentou). Para melhor interpretação da variável alimentação, foi realizada análise de cluster por meio da inclusão de nove questões (consumo de verduras, frutas, feijão, alimentos integrais, presunto, comida congelada, salgadinhos de pacote, chocolate e refrigerante), sendo classificada em melhor e pior padrão alimentar.

Quanto às condições de saúde durante a pandemia, as variáveis apresentadas foram: percepção do estado de saúde (melhorou; ficou igual; piorou), índice de massa corporal IMC (normal; sobrepeso; obesidade), problemas no sono (não afetou, continuou dormindo bem; tinha problemas de sono, mas diminuiu; continuou tendo os mesmos problemas de sono; já tinha problemas de sono e eles pioraram; começou a ter problemas de sono), ansiedade e/ou depressão diagnosticada por médico (não; sim), "Medo da COVID-19"14 (pouco medo; medo moderado; muito medo), algum amigo ou familiar desenvolveu sintomas graves de COVID-19 (não; sim), faz parte do grupo de risco para a COVID-19 (não; sim) e infecção autorrelatada mediante teste por COVID-19 (não; sim).

O IMC foi construído a partir do peso e altura autorrelatado pelos professores, calculado pela fórmula: "IMC = peso $(\mathrm{kg}) /$ estatura $(\mathrm{m})^{2 \prime}$ e classificado com pontos de corte estabelecidos pela Organização Mundial da Saúde (OMS), sendo: peso normal - $\leq 24,9 \mathrm{~kg} / \mathrm{m}^{2}$; sobrepeso - de 25 a $29,9 \mathrm{~kg} / \mathrm{m}^{2}$; e obesidade $-\geq 30 \mathrm{~kg} / \mathrm{m}^{2}$. . A Escala de Medo da COVID-19 (EMC-19) é um instrumento que investiga o medo das pessoas em relação à COVID-19 14. A EMC-19 apresenta sete itens, que são respondidos em uma escala tipo Likert (concordo plenamente; concordo parcialmente; não concordo nem discordo; discordo parcialmente; discordo plenamente). O escore total foi obtido a partir do somatório dos itens (mínimo de sete e máximo de 35 pontos), sendo categorizada de sete a 19 pontos como "pouco medo", de 20 a 26 pontos como "medo moderado" e 27 a 35 pontos como "muito medo".

\section{Análises estatísticas}

Os dados foram organizados, auditados e analisados com o auxílio do programa Statistical Package for Social Sciences (SPSS $)^{\circ}$ versão 22.0. Foram apresentadas frequência simples e prevalência das variáveis referentes ao consumo de bebidas alcoólicas. Quanto às variáveis independentes, 
foram realizadas análises bivariadas por meio da regressão de Poisson, com variância, robusta, apresentando razão de prevalência (RP) bruta, intervalo de confiança de 95\% (IC 95\%) e p-valor. Apenas as variáveis que apresentaram p-valor $\leq$ 0,20 foram selecionadas inicialmente para compor o modelo múltiplo por meio da regressão de Poisson, com variância, robusta. A magnitude das associações foi estimada pela RP ajustada, IC 95\% e nível de significância de 5\% (a < 0,05). Para avaliar a qualidade do modelo, utilizou-se o teste Deviance.

\section{Aspectos éticos}

O projeto foi submetido à apreciação do Comitê de Ética em Pesquisa da Universidade Estadual de Montes Claros (Unimontes) e aprovado com Parecer consubstanciado no 4.200.389/2020. Todos os professores participantes receberam cópia do Termo de Consentimento Livre e Esclarecido e assinalaram "sim" à questão relativa à concordância em participar da pesquisa. A pesquisa também cumpriu com a Resolução no 466/12 do Conselho Nacional da Saúde/Ministério da Saúde, que trata de pesquisa com seres humanos.

\section{RESULTADOS}

O formulário foi acessado por 16.210 professores; desses, 15.641 aceitaram participar da pesquisa, resultando em uma taxa de recrutamento de $96,5 \%$ e taxa de completude de 100\%. Houve participação de professores de 93,2\% do total de cidades mineiras. Entre eles, 81,9\% eram do sexo feminino, 97,4\% tinham até 60 anos e 59,5\% tinham renda familiar entre três e cinco salários mínimos.

Em relação ao consumo de bebidas alcoólicas, no período anterior à pandemia da COVID-19, 53,1\% dos participantes relataram não consumir esse tipo de bebida, enquanto $17,6 \%$ consumiram um a dois dias por semana, e 30,2\% ingeriam entre uma e duas doses por dia (Gráfico 1). Durante a pandemia, 20,2\% relataram ter mantido o consumo e 7,1\% aumentaram o consumo de bebidas alcoólicas (Tabela 1).

Na tabela 2 estão descritas as características dos participantes que apresentaram aumento do consumo de bebidas alcoólicas durante a pandemia. Na análise bivariada, as variáveis sexo masculino, faixa etária de 41-59 anos e até 40 anos, renda familiar maior que dois salários mínimos, sem cônjuge, não realização de trabalho remoto, dificuldade em realizar as atividades, adesão parcial e total ao distanciamento social, tabagismo, desejo de cuidar da aparência física, piora do padrão alimentar, não realização de atividade física, aumento ou redução de atividade de lazer, piora da percepção do estado de saúde, IMC elevado, problemas com o sono, ansiedade/depressão, medo da COVID-19 e amigo/familiar que desenvolveu sintomas graves de COVID-19 estiveram associadas ao aumento do consumo de álcool, com nível de significância de 20\% (Tabela 2).
No modelo ajustado, verificou-se a maior prevalência relacionada ao aumento do consumo de álcool entre os homens $(\mathrm{RP}=1,55)$, naqueles com a faixa etária de até 40 anos ( $R P=$ 3,02 ) e de 41 a 59 anos (RP =2,39), nos que possuíam renda familiar de três a cinco salários mínimos (RP $=1,36)$, seis a nove salários mínimos ( $R P=1,85)$ e 10 ou mais salários mínimos $(R P=2,14)$, e entre aqueles sem cônjuge $(R P=1,31)$ (Tabela 2).

Quanto às condições de trabalho, o maior consumo de bebidas alcoólicas foi observado nos que referiram ter dificuldade em realizar as atividades de trabalho de forma moderada $(R P=1,41)$ e ter muita dificuldade $(R P=1,44)$ e nos insatisfeitos com o trabalho durante a pandemia $(R P=1,71)$ (Tabela 2).

Em relação aos comportamentos/hábitos de vida, observou-se a maior prevalência no consumo de bebida alcoólica, durante a pandemia, entre aqueles que aderiram parcialmente ao distanciamento social $(R P=1,53)$, que relataram tabagismo atual $(R P=2,40)$ e tabagismo no passado $(\mathrm{RP}=1,87)$, que diminuíram o desejo de cuidar da aparência física $(R P=1,21)$, que tiveram piora do padrão alimentar $(R P=1,86)$, que diminuíram as atividade de lazer $(R P=1,16)$ e também entre os que aumentaram as atividades de lazer $(\mathrm{RP}=1,60)$. Fatores associados ao menor consumo de álcool durante a pandemia foram as atividades de lazer inalteradas $(\mathrm{RP}=0,74)$ e a permanência do desejo de cuidar da aparência física $(R P=0,69)$ (Tabela 2).

No que tange às condições de saúde, o aumento da prevalência do consumo do álcool ocorreu entre aqueles que diminuíram os problemas de sono durante a pandemia (RP $=1,97$ ), que continuaram tendo problemas de sono durante a pandemia ( $R P=1,76)$, que pioraram os problemas de sono $(\mathrm{RP}=2,78)$, que começaram a ter problemas de sono $(\mathrm{RP}=$ 2,14 ) e aqueles que tiveram familiar ou amigo que desenvolveram sintomas graves da COVID-19 $(R P=1,19)$. A estatística do teste de Deviance obtido no modelo múltiplo final foi igual a 0,318 ( $p$-valor $=0,844$ ), indicando que o modelo apresentou ajuste adequado (Tabela 2).

\section{DISCUSSÃO}

Os dados deste estudo evidenciaram que, entre os professores da rede estadual de educação básica de Minas Gerais que consumiam bebiba alcoólica antes da pandemia, a maioria manteve o seu consumo ou o diminuiu durante a pandemia (20\% e 13\%, respectivamente), com 7,1\% aumentando o uso e 0,1\% retornando ao consumo de álcool. 0 padrão de aumento do consumo etílico neste estudo não foi o mais frequente. Porém, é importante considerar que a exposição ao álcool é influenciada de acordo com o tipo de substância, frequência e quantidade de uso. O uso abusivo pode levar a doenças físicas e mentais, gerar mortes prematuras, entre outros prejuízos ao longo da vida. 
Tabela 1. Consumo de bebida alcoólica antes e durante a pandemia da COVID-19; Projeto ProfSMoc - Etapa Minas Covid, 2020 ( $n=15.641$ )

\begin{tabular}{|c|c|c|}
\hline Variáveis & $\mathbf{n}$ & $\%$ \\
\hline \multicolumn{3}{|l|}{ Consumo de bebida alcoólica em dias da semana ANTES da pandemia } \\
\hline Não consome bebida alcoólica & 8.308 & 53,1 \\
\hline Menos de uma vez por semana & 4.229 & 27,0 \\
\hline 1 a 2 dias & 2.752 & 17,6 \\
\hline 3 a 4 dias & 271 & 1,7 \\
\hline 5 ou mais dias & 81 & 0,5 \\
\hline \multicolumn{3}{|l|}{ Doses de bebida alcoólica por dia da semana ANTES da pandemia } \\
\hline Não consome bebida alcoólica & 8.468 & 54,1 \\
\hline 1 dose & 2.760 & 17,6 \\
\hline 2 doses & 1.971 & 12,6 \\
\hline 3 doses & 1.092 & 7,0 \\
\hline 4 doses & 627 & 4,0 \\
\hline 5 doses ou mais & 723 & 4,6 \\
\hline \multicolumn{3}{|l|}{ Consumo de bebida alcoólica DURANTE a pandemia } \\
\hline Não consumia bebida alcoólica antes da pandemia e não consumiu durante & 8.052 & 51,5 \\
\hline Não consumiu bebida alcoólica & 1.238 & 7,9 \\
\hline Estava bebendo menos do que costumava & 2.027 & 13,0 \\
\hline Continuou bebendo com a mesma frequência & 3.165 & 20,2 \\
\hline Estava bebendo mais do que costumava & 1.112 & 7,1 \\
\hline Tinha parado de beber, mas voltou a beber novamente & 47 & 0,3 \\
\hline
\end{tabular}

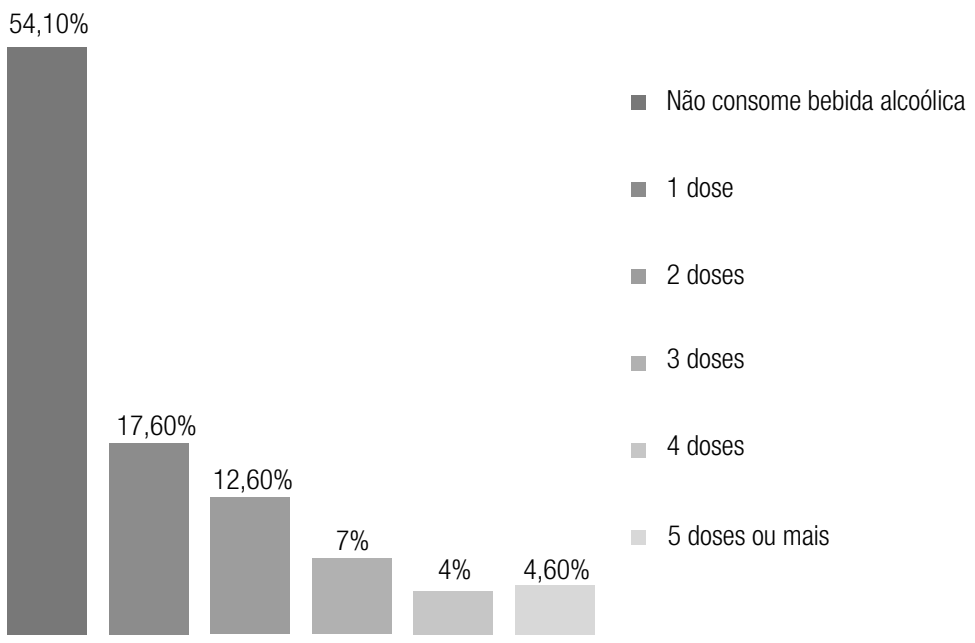

Gráfico 1. Doses de bebida alcoólica por dia da semana ANTES da pandemia da COVID-19; Projeto ProfSMoc - Etapa Minas Covid, 2020 ( $n$ = 15.641)

O aumento do consumo de álcool foi associado a fatores sociodemográficos e econômicos (sexo, idade, renda familiar, situação conjugal), condições de trabalho durante a pandemia (realização de trabalho remoto, dificuldade em realizar as atividades, satisfação com o trabalho), comportamentos/hábitos de vida (adesão ao distanciamento social, tabagismo, desejo de cuidar da aparência física, alimentação, atividade de lazer) e condições de saúde (problemas no sono, algum amigo/familiar desenvolveu sintomas graves de COVID-19). 
Tabela 2. Maior consumo de bebida alcoólica durante a pandemia da COVID-19; regressão de Poisson bruta e ajustada; ProfSMoc - Etapa Minas Covid, 2020 $(n=15.641)$

\begin{tabular}{|c|c|c|c|c|c|}
\hline Variáveis & n (\%) & $\begin{array}{l}\text { RP (IC95\%) } \\
\text { bruta }\end{array}$ & p-valor & $\begin{array}{l}\text { RP (IC95\%) } \\
\text { ajustada }\end{array}$ & p-valor \\
\hline \multicolumn{6}{|l|}{ Perfil sociodemográfico e econômico } \\
\hline Sexo & & & 0,000 & & 0,000 \\
\hline Feminino & $827(6,5)$ & 1,00 & & 1,00 & \\
\hline Masculino & $332(11,8)$ & $1,82(1,61-2,05)$ & & $1,55(1,37-1,76)$ & \\
\hline Idade (anos) & & & 0,000 & & 0,000 \\
\hline 60 ou mais & $9(2,2)$ & 1,00 & & 1,00 & \\
\hline 41 a 59 & $577(6,6)$ & $2,92(1,52-5,60)$ & & $2,39(1,28-4,47)$ & \\
\hline Até 40 & $573(8,9)$ & $3,96(2,06-7,59)$ & & $3,02(1,61-5,64)$ & \\
\hline Renda familiar (salários mínimos) & & & 0,000 & & 0,000 \\
\hline 1 a 2 & $214(5,4)$ & 1,00 & & 1,00 & \\
\hline 3 a 5 & $706(7,6)$ & $1,40(1,21-1,63)$ & & $1,36(1,18-1,57)$ & \\
\hline 6 a 9 & $197(10,1)$ & $1,87(1,56-2,26)$ & & $1,85(1,54-2,23)$ & \\
\hline 10 ou mais & $42(9,9)$ & $1,82(1,33-2,50)$ & & $2,14(1,56-2,92)$ & \\
\hline Situação conjugal & & & 0,000 & & 0,000 \\
\hline Com cônjuge & $681(6,5)$ & 1,00 & & 1,00 & \\
\hline Sem cônjuge & $478(9,2)$ & $1,41(1,26-1,58)$ & & $1,31(1,17-1,46)$ & \\
\hline \multicolumn{6}{|c|}{ Condições de trabalho durante a pandemia } \\
\hline Realização de trabalho remoto & & & 0,075 & & \\
\hline Sim & $1.145(7,4)$ & 1,00 & & - & - \\
\hline Não & $14(11,6)$ & $1,56(0,95-2,57)$ & & - & - \\
\hline Dificuldade de realizar as atividades & & & 0,000 & & 0,015 \\
\hline Nenhuma dificuldade & $55(3,7)$ & 1,00 & & 1,00 & \\
\hline Pouca & $229(5,5)$ & $1,46(1,09-1,95)$ & & $1,20(0,90-1,59)$ & \\
\hline Moderada & $525(7,7)$ & $2,07(1,58-2,72)$ & & $1,41(1,08-1,86)$ & \\
\hline Muita & $350(10,9)$ & $2,92(2,21-3,85)$ & & $1,44(1,08-1,92)$ & \\
\hline Satisfação com o trabalho & & & 0,000 & & 0,000 \\
\hline Satisfeito(a) & $129(3,8)$ & 1,00 & & 1,00 & \\
\hline Nem satisfeito(a) nem insatisfeito(a) & $401(5,7)$ & $1,50(1,23-1,82)$ & & $1,16(0,95-1,41)$ & \\
\hline Insatisfeito(a) & $629(11,9)$ & $3,12(2,59-3,75)$ & & $1,71(1,41-2,08)$ & \\
\hline \multicolumn{6}{|c|}{ Comportamentos/hábitos de vida durante a pandemia } \\
\hline Adesão ao distanciamento social & & & 0,000 & & 0,000 \\
\hline Sim, totalmente & $829(6,6)$ & 1,00 & & 1,00 & \\
\hline Sim, parcialmente & $327(10,6)$ & $1,59(1,40-1,79)$ & & $1,53(1,36-1,73)$ & \\
\hline Não & $3(5,1)$ & $0,76(0,25-2,31)$ & & $0,89(0,28-2,84)$ & \\
\hline Tabagismo & & & 0,000 & & 0,000 \\
\hline Não & $893(6,3)$ & 1,00 & & 1,00 & \\
\hline Ex-fumante & $92(13,8)$ & $2,18(1,79-2,67)$ & & $1,87(1,53-2,28)$ & \\
\hline $\operatorname{Sim}$ & $174(20,4)$ & $3,22(2,78-3,73)$ & & $2,40(2,07-2,78)$ & \\
\hline Desejo de cuidar da aparência física & & & 0,000 & & 0,000 \\
\hline Aumentou & $171(7,7)$ & 1,00 & & 1,00 & \\
\hline Permaneceu o mesmo & $338(4,3)$ & $0,56(0,47-0,67)$ & & $0,69(0,58-0,83)$ & \\
\hline Diminuiu & $650(11,6)$ & $1,50(1,27-1,76)$ & & $1,21(1,03-1,44)$ & \\
\hline Alimentação & & & 0,000 & & 0,000 \\
\hline Melhor padrão alimentar & $327(4,2)$ & 1,00 & & 1,00 & \\
\hline Pior padrão alimentar & $832(10,5)$ & $2,49(2,20-2,81)$ & & $1,86(1,64-2,10)$ & \\
\hline
\end{tabular}




\begin{tabular}{|c|c|c|c|c|c|}
\hline Variáveis & n (\%) & $\begin{array}{l}\text { RP (IC95\%) } \\
\text { bruta }\end{array}$ & p-valor & $\begin{array}{l}\text { RP (IC95\%) } \\
\text { ajustada }\end{array}$ & p-valor \\
\hline Prática de atividade física & & & 0,000 & & \\
\hline $\operatorname{Sim}$ & $589(6,7)$ & 1,00 & & - & - \\
\hline Não & $570(8,3)$ & $1,24(1,11-1,39)$ & & - & - \\
\hline Atividade de lazer & & & 0,000 & & 0,000 \\
\hline Não estava realizando atividades de lazer & $507(7,5)$ & 1,00 & & 1,00 & \\
\hline Diminuiu & $444(8,8)$ & $1,17(1,03-1,32)$ & & $1,16(1,03-1,31)$ & \\
\hline Não se alterou & $113(3,8)$ & $0,49(0,40-0,60)$ & & $0,74(0,60-0,90)$ & \\
\hline Aumentou & $95(10,8)$ & $1,43(1,16-1,77)$ & & $1,60(1,31-1,97)$ & \\
\hline \multicolumn{6}{|l|}{ Condições de saúde durante a pandemia } \\
\hline Percepção do estado de saúde & & & 0,000 & & \\
\hline Melhorou & $42(5,6)$ & 1,00 & & - & - \\
\hline Ficou igual & $319(4,1)$ & $0,72(0,53-0,99)$ & & - & - \\
\hline Piorou & $798(11,3)$ & $2,00(1,48-2,70)$ & & - & - \\
\hline $\mathrm{IMC}^{*+}$ & & & 0,001 & & \\
\hline Normal & $490(6,7)$ & 1,00 & & - & - \\
\hline Sobrepeso & $341(7,8)$ & $1,16(1,01-1,33)$ & & - & - \\
\hline Obesidade & $320(8,7)$ & $1,30(1,13-1,48)$ & & - & - \\
\hline Problemas no sono & & & 0,000 & & 0,000 \\
\hline Não afetou, continuou dormindo bem & $213(3,2)$ & 1,00 & & 1,00 & \\
\hline Tinha problemas de sono, mas diminuiu & $11(8,0)$ & $2,45(1,37-4,39)$ & & $1,97(1,12-3,46)$ & \\
\hline Continuou tendo os mesmos problemas de sono & $142(7,3)$ & $2,23(1,81-2,74)$ & & $1,76(1,43-2,16)$ & \\
\hline Já tinha problemas de sono e eles pioraram & $269(15,2)$ & $4,68(3,94-5,56)$ & & $2,78(2,31-3,34)$ & \\
\hline Começou a ter problemas de sono & $524(10,0)$ & $3,08(2,64-3,60)$ & & $2,14(1,83-2,51)$ & \\
\hline Ansiedade e/ou depressão & & & 0,000 & & \\
\hline Não & $765(6,6)$ & 1,00 & & - & - \\
\hline Sim & $394(9,7)$ & $1,47(1,31-1,65)$ & & - & - \\
\hline \multicolumn{6}{|l|}{ Medo da COVID-19 } \\
\hline Pouco medo & $233(5,3)$ & 1,00 & & - & - \\
\hline Medo moderado & $311(7,0)$ & $1,31(1,11-1,54)$ & & - & - \\
\hline Muito medo & $615(9,0)$ & $1,68(1,45-1,95)$ & & - & - \\
\hline $\begin{array}{l}\text { Algum amigo ou familiar desenvolveu sintomas graves } \\
\text { de COVID-19 }\end{array}$ & & & 0,000 & & 0,001 \\
\hline Não & $625(6,3)$ & 1,00 & & 1,00 & \\
\hline $\operatorname{Sim}$ & $534(9,5)$ & $1,51(1,35-1,68)$ & & $1,19(1,07-1,32)$ & \\
\hline Faz parte do grupo de risco para a COVID-19 & & & 0,024 & & \\
\hline Não & $709(7,1)$ & 1,00 & & - & - \\
\hline $\operatorname{Sim}$ & $450(8,0)$ & $0,87(0,78-0,98)$ & & - & - \\
\hline Infecção por COVID-19 & & & 0,809 & & \\
\hline Não & $1.146(7,4)$ & 1,00 & & - & - \\
\hline Sim & $13(7,0)$ & $0,93(0,55-1,58)$ & & - & - \\
\hline Deviance: 0,318 / $p$-valor: 0,844 & & & & & \\
\hline
\end{tabular}

RP: razão de prevalência; IC95\%: intervalo de confiança de 95\%; p-valor: Teste de Wald; IMC: índice de massa corporal. *Variação no $n$ devido à perda de informação.

+Foram desconsideradas da análise as mulheres grávidas $(n=246)$. 
Neste estudo, parcela considerável dos docentes consumiu álcool durante a pandemia. Esse resultado está em consonância com os de outros estudos realizados na população adulta durante o distanciamento social da pandemia, o que pode ser justificado pelos impactos psicológicos causados pelo contexto atual ${ }^{1,16,17}$. Na população belga, o aumento do uso de bebidas alcoólicas, nesse contexto, foi explicado devido a maior tempo livre, tédio e preocupações com o contexto mundial e como forma de relaxamento ${ }^{16}$. Destaque importante durante a pandemia é a probabilidade de o uso de bebidas alcoólicas agravar infecções de COVID-19, com lesões pulmonares e alterações no sistema imune e piora de outras condições médicas gerais e psiquiátricas ${ }^{18,19}$.

\section{Perfil sociodemográfico e econômico}

Neste estudo, verificou-se que o aumento do consumo de álcool apresentou maior prevalência no sexo masculino. Esse resultado se assemelha ao encontrado na $\mathrm{China}^{20}$ e na Bélgica ${ }^{16}$, em que a maioria dos usuários dessa substância era do sexo masculino, apresentando maior risco de abuso. Estudo realizado em todas as unidades da federação brasileira durante a pandemia, na população geral maior de 18 anos, não encontrou diferença estatística significativa do consumo de álcool relacionado ao sexo'. A literatura apresenta que a ingestão elevada de bebidas alcoólicas nos homens se explica por fatores biológicos, hormonais e psicossociais, farmacocinética do álcool e interações com a função cerebral21, sendo os homens propensos a esse comportamento devido à pressão social para maior ingestão etílica ${ }^{20}$.

Observou-se uma maior prevalência do aumento do consumo de bebidas alcoólicas entre professores na faixa etária de 41-59 anos e até 40 anos. Em estudos internacionais e nacionais, realizados durante a pandemia, em população geral de adultos, também se observou aumento do uso do álcool, principalmente entre a população mais jovem ${ }^{1,22,23}$. Durante a pandemia, os mais jovens foram o grupo mais propenso a ter problemas psicológicos, como ansiedade e depressão, associados à redução do bem-estar mental, com consequente aumento do uso de álcool ${ }^{22}$. A população mais velha é mais estável à percepção do estresse agudo do distanciamento social e da pandemia COVID-19 ${ }^{16}$.

Neste estudo, os professores com renda familiar maior que dois salários mínimos estiveram associados ao maior consumo de bebidas alcoólicas. No Brasil, contudo, durante a pandemia, estudo comportamental em todo o território nacional, realizado na população geral, demonstrou a redução dos níveis socioeconômicos, com a queda da renda familiar24. O maior uso do álcool provavelmente pode ser explicado devido ao maior poder aquisitivo para a compra da bebida e ao estilo de vida, com essa substância apresentando menor estigma em relação às demais ${ }^{25}$.

Os docentes sem cônjuge apresentaram maior prevalência de aumento no consumo de álcool. Dados similares foram encontrados em estudo conduzido na Alemanha, na população geral, verificando-se que pessoas casadas ou com companheiro(a) apresentaram menores níveis de estresse, enquanto pessoas solteiras ou que moravam sozinhas apresentaram maior sofrimento menta ${ }^{26}$. As situações de pandemia foram consideradas como eventos estressores e relacionadas com o aumento do uso de álcool entre solteiros ${ }^{27}$.

\section{Condições de trabalho durante a pandemia}

O desfecho investigado associou-se ao fato de os docentes terem moderada ou muita dificuldade com as atividades de trabalho e estarem insatisfeitos com ele 1 . Nesse contexto da COVID-19, as atividades remotas passaram a ser a estratégia utilizada para o processo de ensino-aprendizagem, com um quarto dos professores alterando as circunstâncias do trabaIho para o cenário doméstico. Isso foi associado a maior estresse, principalmente devido ao acúmulo de atividades domésticas e laborais, assim como ao distanciamento social ${ }^{24}$.

\section{Comportamentos/hábitos de vida durante a pandemia}

Os docentes que aderiram ao distanciamento social estiveram associados ao maior consumo etílico durante a pandemia. Estudo em população geral chinesa, em Hubei, demonstrou maior suscetibilidade a agravos de doenças mentais e aumento do uso de álcool associados à pande$\mathrm{mia}^{22}$. Resultados semelhantes foram encontrados em estudos em território nacional ${ }^{1,28,29}$, o que provavelmente explica o estresse psicológico do distanciamento social associado a esse aumento do consumo etílico.

O tabagismo atual ou prévio foi associado à ingestão aumentada de bebida alcoólica neste estudo. Estudo realizado na Bélgica demonstrou aumento do tabagismo e uso do álcool durante a pandemia, ambos associados ao tédio, ao convívio e a eventos estressores ${ }^{30}$. Resultado semelhante foi verificado em estudo nacional, na população geral, que associa a pandemia da COVID-19 à piora dos comportamentos de riscos (tabagismo, etilismo) e situações emocionais instáveis'. Esses fatores são preocupantes para a saúde pública, devido ao fato de o tabagismo interferir em fatores de risco pulmonares e cardiovasculares, com potencial de agravo dos sintomas da COVID-19'18. O tabagismo atual foi associado a pior probabilidade de efeitos adversos em relação aos que já cessaram o hábito, neste contexto da pandemia ${ }^{31}$.

No presente estudo, a variável desejo de cuidar da aparência física esteve associada ao desfecho estudado. A permanência inalterada desses cuidados associou-se como fator protetor ao uso de bebidas alcoólicas, enquanto a redução associou-se ao aumento do consumo etílico. Estudo prévio à pandemia, em idosas brasileiras, já associava insatisfação da aparência física à piora da qualidade de vida ${ }^{32}$. Neste conturbado momento histórico, essas associações podem se traduzir como reflexo da saúde física e mental ${ }^{1,16,17}$, 
gerando consequências no aumento do uso de álcool entre os docentes.

No que concerne à alimentação dos docentes, houve uma associação significativa entre a piora desse fator e o consumo aumentado de bebidas alcoólicas. Estudo realizado no Brasil demonstrou piora do padrão alimentar durante a pandemia, com redução da ingesta de verduras/frutas e crescente consumo de alimentos processados' ${ }^{1}$. A pandemia gerou agravos psicológicos, piora da alimentação e aumento do consumo de álcool33, conforme se verificou em jovens egípcios o aumento da alimentação relacionada ao estado emocional ${ }^{34}$. Destaca-se a relevante influência do sobrepeso e da obesidade nos agravos da COVID-1935.

As atividades de lazer, quando inalteradas, foram associadas ao menor consumo de álcool. No entanto, ter aumentado ou diminuído essas atividades relacionou-se à elevação do consumo de bebidas etílicas. Estudo com jovens brasileiros e portugueses, antes da pandemia, já demonstrava que a maioria dos participantes consumia álcool nos momentos de tempo livre, associado às atividades de lazer (sociais, esportivas e solitárias) ${ }^{36}$. Na atual pandemia, as alterações comportamentais podem ser relacionadas à piora do bem-estar físico e mental, aumentando-se o consumo etílico; esses fatores podem representar situações com potenciais de gravidade da COVID-19'.

Ter familiar ou amigo que desenvolveu sintomas graves de COVID-19 associou-se a maior consumo de bebida alcoólica. Esse resultado assemelha-se ao de um estudo italiano que evidenciou aumento do estresse e uso de bebidas alcoólicas nos participantes que tiveram o diagnóstico de COVID-19 ou que tiveram familiar e/ou amigo com a doença, representando o agravo de problemas psicológicos e o consumo de bebida alcoólica durante a pandemia ${ }^{17}$.

\section{Condições de saúde durante a pandemia}

No presente estudo, ter problemas relacionados ao sono foi associado à elevação do consumo de bebidas alcoólicas. A piora na qualidade do sono foi relatada em associação à piora dos comportamentos não saudáveis durante a pandemia da COVID-19, em pesquisa realizada em todo o território brasileiro ${ }^{37}$. A redução da qualidade de sono provoca impactos negativos na saúde menta| ${ }^{38}$, o que pode explicar o comportamento de maior consumo de bebida alcoólica entre professores.

O presente estudo apresenta algumas limitações, principalmente relacionadas ao processo de obtenção de dados pela internet como método exclusivo, com probabilidade de vieses de seleção e de memória e utilização de valores autorreferidos de peso e estatura para o IMC. No entanto, os valores antropométricos autorreferidos são uma forma simples, de baixo custo e que se aplica a estudos de grandes grupos populacionais ${ }^{39,40}$. Deve-se considerar que, por se tratar de um estudo transversal, não é possível estabelecer relações de causalidade. Destacam-se os pontos fortes deste estudo, como metodologia rigorosa, população amostral robusta e bem distribuída nas diversas regiões do estado e importante apoio da SEE-MG.

\section{CONCLUSÃO}

Os resultados deste estudo evidenciaram que a maioria dos participantes manteve o seu consumo ou o diminuiu durante a pandemia (20\% e 13\%, respectivamente), com 7,1\% aumentando o uso e 0,1\% retornando ao consumo de álcool. O aumento do consumo etílico foi associado a fatores sociodemográficos e econômicos (sexo masculino, idade até 60 anos, renda familiar maior que dois salários mínimos, sem cônjuge), condições de trabalho durante a pandemia (não realização de trabalho remoto, dificuldade em realizar as atividades, insatisfação com o trabalho), comportamentos/ hábitos de vida (adesão parcial ao distanciamento social, tabagismo atual e prévio, redução do desejo de cuidar da aparência física, piora do padrão alimentar, redução e aumento da atividade de lazer) e condições de saúde (problemas com o sono, algum amigo/familiar desenvolveu sintomas graves de COVID-19).

O consumo de álcool entre os docentes, durante a epidemia do COVID-19, pode ter impactos na saúde mental e física dos professores, associado às recentes mudanças no processo de trabalho docente. Políticas públicas no setor da educação e promoção no cuidado da saúde mental refletem necessidades atuais e urgentes, como forma de proteção a esse trabalhador e seus vínculos sociais.

\section{CONTRIBUIÇÕES INDIVIDUAIS}

Ana Cláudia Alves Leão - Contribuiu para a concepção, design, análise e interpretação dos dados; contribuiu para redigir o artigo e revisá-lo para conteúdo intelectual importante; deu a aprovação final da versão a ser publicada.

Nayra Suze Souza e Silva - Contribuiu para a concepção, design, análise e interpretação dos dados; contribuiu para redigir o artigo e revisá-lo para conteúdo intelectual importante; deu a aprovação final da versão a ser publicada.

Romerson Brito Messias - Contribuiu para a concepção, design, análise e interpretação dos dados; contribuiu para redigir o artigo e revisá-lo para conteúdo intelectual importante; deu a aprovação final da versão a ser publicada.

Desirée Sant'Ana Haikal - Contribuiu para a concepção, design, análise e interpretação dos dados; contribuiu para redigir o artigo e revisá-lo para conteúdo intelectual importante; deu a aprovação final da versão a ser publicada. 
Marise Fagundes Silveira - Contribuiu para a concepção, design, análise e interpretação dos dados; contribuiu para redigir o artigo e revisá-lo para conteúdo intelectual importante; deu a aprovação final da versão a ser publicada.

Rosângela Ramos Veloso Silva - Contribuiu para a concepção, design, análise e interpretação dos dados; contribuiu para redigir o artigo e revisá-lo para conteúdo intelectual importante; deu a aprovação final da versão a ser publicada.

Maria Fernanda Santos Figueiredo Brito - Contribuiu para a concepção, design, análise e interpretação dos dados; contribuiu para redigir o artigo e revisá-lo para conteúdo intelectual importante; deu a aprovação final da versão a ser publicada.

\section{CONFLITO DE INTERESSES}

Todos os autores declararam não apresentar conflito de interesses.

\section{AGRADECIMENTOS}

Agradecemos aos professores do estado de Minas Gerais, por participarem do Projeto ProfSMoc - Etapa Minas Covid, à Unimontes e à SEE-MG, pelo apoio, e à Coordenação de Aperfeiçoamento de Pessoal de Nível Superior (Capes), pela concessão de bolsas.

\section{REFERÊNCIAS}

1. Malta DC, Szwarcwald CL, Barros MBZ, Gomes CS, Machado IE, Souza Junior PRB, et al. A pandemia da COVID-19 e as mudanças no estilo de vida dos brasileiros adultos: um estudo transversal, 2020. Epidemiol Serv Saúde. 2020;29(4):e2020407.

2. Sallie SN, Ritou V, Bowden-Jones H, Voon V. Assessing international alcohol consumption patterns during isolation from the COVID-19 pandemic using an online survey: highlighting negative emotionality mechanisms. BMJ Open. 2020;10:e044276.

3. Valente JY, Sohi I, Garcia-Cerde R, Monteiro MG, Sanchez ZM. What is associated with the increased frequency of heavy episodic drinking during the COVID-19 pandemic? Data from the PAHO regional web-based survey. Drug Alcohol Depend. 2021;221:108621.

4. Garnett C, Jackson S, Oldham M, Brown J, Steptoe A, Fancourt D. Factors associated with drinking behaviour during COVID-19 social distancing and lockdown among adults in the UK. Drug Alcohol Depend. 2021;219:108461.

5. Pollard MS, Tucker JS, Green HD. Changes in adult alcohol use and consequences during the COVID-19 pandemic in the US. JAMA. 2020;3(9):e2022942.

6. Koopmann A, Georgiadou E, Kiefer F, Hillemacher T. Did the general population in Germany drink more alcohol during the COVID-19 pandemic lockdown? Alcohol Alcoholism. 2020;55(6):698-9.

7. Peacock A, Leung J, Larney S, Colledge S, Hickman M, Rehm J, et al. Global statistics on alcohol, tobacco and illicit drug use: 2017 status report. Addiction. 2018;113(10):1905-26.

8. Abreu MNS, Eleotério AE, Oliveira FA, Pedroni LCBR, Lacena EE. Prevalência e fatores associados ao consumo excessivo episódico de álcool entre adultos jovens brasileiros de 18 a 24 anos. Rev Bras Epidemiol. 2020;23:e200092.

9. Columb D, Hussain R, O'Gara C. Addiction psychiatry and COVID-19: impact on patients and service provision. Ir J Psychol Med. 2020;37(3):164-8.
10. Garcia LP, Sanchez ZM. Consumo de álcool durante a pandemia da COVID-19: uma reflexão necessária para o enfrentamento da situação. Cad Saúde Pública. 2020;36(10):e00124520.

11. Souza KR, Borges SG, Rodrigues AMS, Feliz EG, Gomes L, Rocha GL, et al. Trabalho remoto, saúde docente e greve virtual em cenário de pandemia. Trab Educ Saúde. 2021;19:e00309141.

12. Secretaria de Estado de Educação de Minas Gerais. Relação de estabelecimentos de ensino ativos em Minas Gerais. Disponível em: https://www2.educacao.mg.gov.br/mapa-dosite/parceiro/lista-de-escolas. 2020. Acesso em: 15 fev. 2021.

13. Eysenbach G. Improving the quality of Web surveys: the Checklist for Reporting Results of Internet E-Surveys (CHERRIES). J Med Internet Res. 2004;6(3):e34.

14. Ahorsu DK, Lin CY, Imani V, Saffari M, Griffiths MD, Pakpour AH. O Medo da Escala COVID-19: Desenvolvimento e Validação Inicial. Rev Int Saúde Ment Vício. 2020;1-9.

15. World Health Organization (WHO). Physical status: the use and interpretation of anthropometry. Report of a WHO Expert Committee. Geneva: WHO; 1995.

16. Schmits E, Glowacz F. Changes in Alcohol Use During the COVID-19 Pandemic: Impact of the Lockdown Conditions and Mental Health Factors. Int J Ment Health Addict. 2021;1-12.

17. Panno A, Carbone GA, Massullo C, Farina B, Imperatori C. COVID-19 Related Distress is Associated with Alcohol Problems, Social Media and Food Addiction Symptoms: Insights from the Italian Experience During the Lockdown. Front Psychiatry. 2020;11:e577135.

18. Althobaiti YS, Alzahrani MA, Alsharif NA, Alrobaie NS, Alsaab HO, Uddin MN. A possivel relação entre 0 abuso de tabaco, opioide ou álcool com COVID-19. Saúde (Basileia). 2020;9(1):2.

19. Collins SE. Associations Between Socioeconomic Factors and Alcohol Outcomes. Álcool Res. 2016;38(1):83-94.

20. Wang Y, Lu H, Hu M, Wu S, Chen J, Wang L, et al. Alcohol Consumption in China Before and During COVID-19: Preliminary Results From an Online Retrospective Survey. Front Psychiatry. 2020;11:e597826.

21. Erol A, Karpyak VM. Sex and gender-related differences in alcohol use and its consequences: Contemporary knowledge and future research considerations. Drug Alcohol Depend. 2015;156:1-13

22. Ahmed MZ, Ahmed O, Aibao Z, Hanbin S, Siyu L, Ahmad A. Epidemic of COVID-19 in China and associated Psychological Problems. Asian J Psychiatr. 2020;51:e102092.

23. Jacob L, Smith L, Armstrong NC, Yakkundi A, Barnett Y, Butler L, et al. Alcohol use and mental health during COVID-19 lockdown: A cross-sectional study in a sample of UK adults. Drug Alcohol Depend. 2021;219:e108488.

24. Almeida WS, Szwarcwald CL, Malta DC, Barros MBA, Souza Junior PRB, Azevedo LO, et al. Mudanças nas condições socioeconômicas e de saúde dos brasileiros durante a pandemia de COVID-19. Rev Bras Epidemiol. 2020;23:e200105.

25. Rolland B, Haesebaert F, Zante E, Benyamina A, Haesebaert J, Franck N. Global Changes and Factors of Increase in Caloric/Salty Food Intake, Screen Use, and Substance Use During the Early COVID-19 Containment Phase in the General Population in France: Survey Study. JMIR Public Health Surveill. 2020;6(3):e19630.

26. Schnell T, Krampe H. Meaning in Life and Self-Control Buffer Stress in Times of COVID-19: Moderating and Mediating Effects with Regard to Mental Distress. Front Psychiatry. 2020;11:e582352.

27. Gonçalves PD, Moura HF, Amaral RA, Castaldelli-Maia JM, Malbergier A. Alcohol Use and COVID-19: Can we Predict the Impact of the Pandemic on Alcohol Use Based on the Previous Crises in the 21st Century? A Brief Review. Front Psychiatry. 2020;11:e581113.

28. Pereira MD, Oliveira LC, Costa CFT, Bezerra CMO, Pereira MD, Santos CKA, etal. The COVID-19 pandemic, social isolation, consequences on mental health and coping strategies: an integrative review. Res Soc Dev. 2020;9(7):1-35.

29. Szwarcwald CL, Souza Júnior PRB, Malta DC, Barros MBZ, Magalhães MAFM, Xavier DR, et al. Adesão às medidas de restrição de contato físico e disseminação da COVID-19 no Brasil. Epidemiol Serv Saúde. 2020;29(5):e2020432.

30. Vanderbruggen N, Matthys F, Van Laere S, Zeeuws D, Santermans L, Van den Ameele S, et al. Self-Reported Alcohol, Tobacco, and Cannabis Use during COVID-19 Lockdown Measures: Results from a Web-Based Survey. Eur Addict Res. 2020;26(6):309-15.

31. Farsalinos K, Barbouni A, Poulas K, Polosa R, Caponnetto P, Niaura R. Current smoking, former smoking, and adverse outcome among hospitalized COVID-19 patients: a systematic review and meta-analysis. Ther Adv Chronic Dis. 2020;11:2040622320935765. 
32. Skopinski F, Resende TL, Schneider RH. Imagem corporal, humor e qualidade de vida. Rev Bras Geriatr Gerontol. 2015;18(1):95-105.

33. Kriaucioniene V, Bagdonaviciene L, Rodríguez-Pérez C, Petkeviciene J. Associations between Changes in Health Behaviors and Body Weight during the COVID-19 Quarantine in Lituânia: The Litun COVIDiet Study. Nutrientes. 2020;12(10):e3119.

34. Alamrawy RG, Fadl N, Khaled A. Psychiatric morbidity and dietary habits during COVID-19 pandemic: a cross-sectional study among Egyptian Youth (14-24 years). Middle East Curr Psychiatry. 2021;28(1):6.

35. Simonnet A, Chetboun M, Poissy J, Raverdy V, Noulette J, Duhamel A, et al.; LICORN and the Lille COVID-19 and Obesity. High Prevalence of Obesity in Severe Acute Respiratory Syndrome Coronavirus-2 (SARS-CoV-2) Requiring Invasive Mechanical Ventilation. Obesity (Silver Spring). 2020;28(7):1195-9.

36. Romera L, Martins R, Heringer Freitas, H, Dos D, Tinôco D, Rondina R. Tempo livre e uso de álcool e outras drogas: estudo comparativo entre estudantes universitários do Brasil e Portugal. Movimento: Rev Esc Educ Fís. 2018;24(3):765-76.
37. Werneck A0, Silva DR, Malta DC, Lima MG, Souza-Júnior PRB, Azevedo LO, et al. The mediation role of sleep quality in the association of the incidence of unhealthy movement behaviors due to COVID-19 quarantine and mental health. Sleep Med. 2020;76:10-5.

38. Barros MBA, Lima MG, Malta DC, Szwarwald CL, Azevedo RCS, Romero D, et al. Relato de tristeza/depressão, nervosismo/ansiedade e problemas de sono na população adulta brasileira durante a pandemia de COVID-19. Epidemiol Serv Saúde. 2020;29(4):e2020427.

39. Silveira EA, Araújo CL, Gigante DP, Barros AJD, Lima MS. Validação do peso e altura referidos para o diagnóstico do estado nutricional em uma população de adultos no sul do Brasil. Cad Saúde Pública. 2005;21(1):235-45.

40. Schmidt MI, Duncan BB, Tavares M, Polanczyk CA, Pellanda L, Zimmer PM. Validity of selfreported weight - a study of urban Brazilian adults. Rev Saúde Pública. 1993;27(4):271-6. 